\title{
Klassifikation subaxialer Halswirbelsäulenverletzungen
}

\author{
Clemens Schopper, Markus Schultheiss
}

\section{Einleitung}

Die Klassifikation subaxialer Verletzungen der Halswirbelsäule stellt bis heute eine kontroverse Fragestellung dar, bis dato hat sich kein internationaler Konsens zur einheitlichen Klassifikation etabliert [2]. Die ursprüngliche Klassifikation nach Magerl et al. aus dem Jahr 1994 [1] zur Definition thorakolumbaler, ossärer Verletzungen anhand des Mechanismus (Kompression, Flexion/Distraktion, Rotation) diente dabei stets als morphologische Ausgangsbasis einer erweiterten, differenzierten Klassifikation der Verletzungen im Bereich der Halswirbelsäule.

Lee et al. [4] integrierten in der Beschreibung des TLICS (Thoracolumbar Injury Classification and Severity Score, - Tab. 1) auch den Zustand des posterioren Bandkomplexes. Der TLICS zieht 3 Hauptkriterien zur Beurteilung heran, namentlich die Frakturmorphologie, den Zustand des posterioren Bandkomplexes und den neurologischen Status des Patienten. Auch anhand dieser Klassifikation

- Tab. 1 TLICS-Klassifikation.

\begin{tabular}{|l|c|}
\hline Parameter & Punkte \\
\hline Morphologie & \\
\hline Kompression & 1 \\
\hline Berstung & 2 \\
\hline Rotation/Translation & 3 \\
\hline Distraktion & 4 \\
\hline neurologischer Status & \\
\hline intakt & 0 \\
\hline Wurzelläsion & 2 \\
\hline inkomplette Querschnittläsion & 3 \\
\hline komplette Querschnittläsion & 2 \\
\hline Cauda-equina-Läsion & 3 \\
\hline dorsaler Bandapparat & \\
\hline intakt & 2 \\
\hline Verletzungsverdacht/unklar & 3 \\
\hline verletzt & \\
\hline $\begin{array}{l}\text { Quelle: Patel et al. Subaxial cervical spine trauma classifica- } \\
\text { plen: the Subaxial Injury Classification system and case exam- }\end{array}$ \\
\hline \begin{tabular}{l} 
inosurg Focus 2008; 25: E8 \\
\hline
\end{tabular}
\end{tabular}

wird ein summativer Wert errechnet, der bei der Entscheidungsfindung über konservatives oder operatives Vorgehen behilflich sein soll ( $<4$ konservativ, 4 konservativ/operativ, $\geq 5$ operativ).

Während die ersten Systeme (Allen et al., Harris et al., White et Panjabi) zur Klassifikation zervikaler Wirbelsäulenverletzungen praktisch ausschließlich auf morphologischen Kriterien der Frakturentstehung beruhten [3], haben Patel et al. im Jahr 2008 durch Veröffentlichung der SLIC (Subaxial Injury Classification, - Tab. 2) eine auf 3 Säulen beruhende Einteilung eingeführt, welche auch an der Halswirbelsäule neben der Frakturmorphologie den Zustand des diskoligamentären Komplexes und den neurologischen Status des Patienten berücksichtigt.

Ebenso wird anhand dieser Klassifikation ein summativer Wert errechnet, der bei der Entscheidungsfindung über konservatives oder operatives Vorgehen behilflich sein soll (<4 konservativ, 4 konservativ/operativ, $\geq 5$ operativ).

Tab. 2 SLIC-Klassifikation.

\begin{tabular}{|l|c|}
\hline Parameter & Punkte \\
\hline Morphologie & 0 \\
\hline unauffällig & 1 \\
\hline Kompression & $1-2$ \\
\hline Berstung & 3 \\
\hline Distraktion & 4 \\
\hline Rotation/Translation & \\
\hline diskoligamentärer Komplex & 0 \\
\hline intakt & 1 \\
\hline konventionell-radiografisch nicht offensichtlich & 2 \\
\hline verletzt & \\
\hline neurologischer Status & 0 \\
\hline intakt & 1 \\
\hline Wurzelläsion & 2 \\
\hline komplette Querschnittläsion & 3 \\
\hline inkomplette Querschnittläsion & +1 \\
\hline fortschreitende Myelonkompression & \\
\hline $\begin{array}{l}\text { Quelle: Lee et al. Thoracolumbar injury classification and se- } \\
\text { lumbar spine trauma. J Orthop Sci 2005; 10: 671-675 }\end{array}$ \\
\hline inco-
\end{tabular}




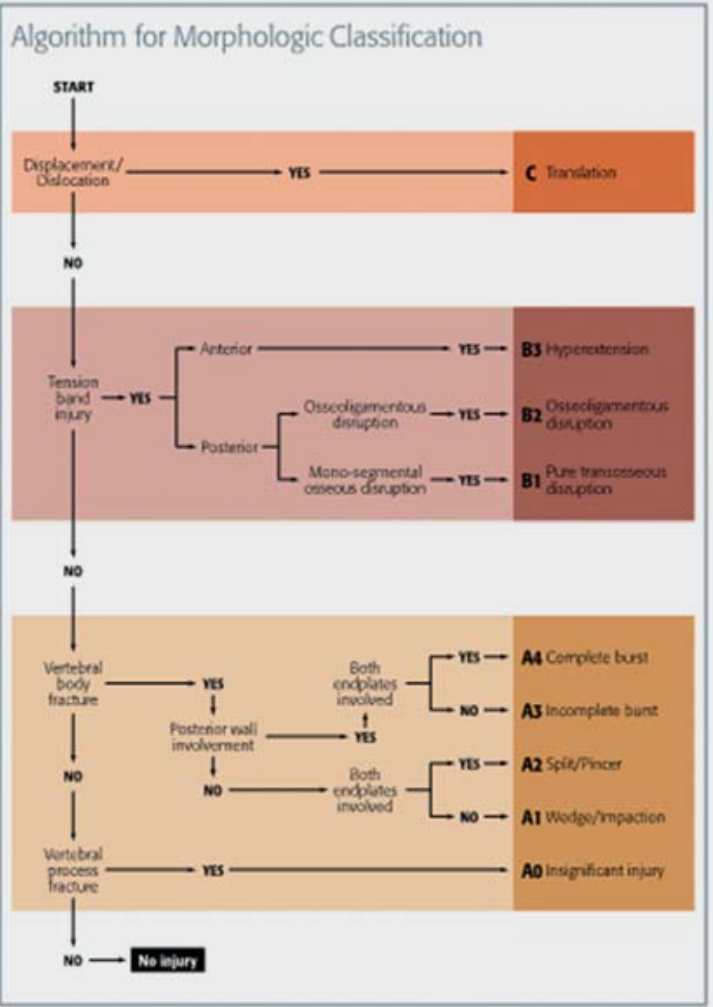

- Abb. 1 Algorithmus der AOSpine-Klassifikation subaxiale HWS: Morphologische Klassifizierung. Copyright by AOSpine International, Switzerland. Quelle: AOFoundation. AOSpine Injury Classification Systems. Im Internet: https://aospine.aofoundation.org/Structure/education/ online-education/classifications/Pages/classifications. aspx; Stand: 08.03.2018 [rerif].

Ausgehend von dieser Entwicklung der Klassifikation zervikaler Wirbelsäulenverletzungen hat es sich die Expertengruppe der AOSpine um Vaccaro et al. [2] zur Aufgabe gemacht, eine Klassifikation für subaxiale knöcherne Verletzungen der Halswirbelsäule zu entwickeln, die sich zwar ebenfalls am morphologischen Grundsatz der AOKlassifikation der Wirbelverletzungen der thorakolumbalen Region orientiert, darüber hinaus aber Elemente der weiterentwickelten Klassifikationen berücksichtigt und neue Aspekte beleuchtet, um der speziellen Anatomie der Halswirbelsäule gerecht zu werden. Im Rahmen der neuen Klassifikationsmethode werden neben den bereits beschriebenen Faktoren folgende zusätzliche Parameter definiert:

- Beteiligung der Facettengelenke

- isolierte Verletzungen ligamentärer Strukturen

- Begleiteinflussfaktoren (Bandscheibenläsionen/qualitative Veränderungen der Knochensubstanz)

Die Anwendung der beschriebenen Kriterien soll eine weitere Verbesserung der Feindifferenzierung des Verletzungsmusters ermöglichen.
- Tab. 3 AOSpine-Klassifikation subaxiale HWS: Typ-AVerletzungen: Kompressionsverletzungen.

\begin{tabular}{|l|l|}
\hline A & Kompressionsverletzungen \\
\hline 0 & $\begin{array}{l}\text { keine oder isolierte, nicht stabilitätsgefährdende } \\
\text { knöcherne Verletzung der hinteren Säule (isolierte } \\
\text { Bogen- oder Dornfortsatzfraktur) }\end{array}$ \\
\hline 1 & $\begin{array}{l}\text { Kompressionsbruch, eine Grund- oder Deckplatte } \\
\text { betreffend ohne Beteiligung der hinteren Wirbel- } \\
\text { körperwand }\end{array}$ \\
\hline 2 & $\begin{array}{l}\text { koronare Spalt- oder Zangenbrüche, beide End- } \\
\text { platten betreffend ohne Beteiligung der hinteren } \\
\text { Wirbelkörperwand }\end{array}$ \\
\hline 3 & $\begin{array}{l}\text { Berstungsfraktur einer Grund- oder Deckplatte mit } \\
\text { Beteiligung der hinteren Wirbelkörperwand }\end{array}$ \\
\hline 4 & $\begin{array}{l}\text { Berstungsbruch oder sagittaler Spaltbruch beide } \\
\text { Endplatten betreffend }\end{array}$ \\
\hline
\end{tabular}

- Tab. 4 AOSpine-Klassifikation subaxiale HWS: Typ-BVerletzungen: Distraktionsverletzungen.

\begin{tabular}{|l|l|}
\hline B & Distraktionsverletzungen \\
\hline 1 & $\begin{array}{l}\text { rein knöcherne Verletzungen im Verlauf des hinte- } \\
\text { ren Längsbandes }\end{array}$ \\
\hline 2 & $\begin{array}{l}\text { komplette Unterbrechung der kapsuloligamentä- } \\
\text { ren hinteren Strukturen ggf. zusammen mit Verlet- } \\
\text { zungen des Wirbelkörpers/der Bandscheibe oder } \\
\text { der Facettengelenke }\end{array}$ \\
\hline 3 & $\begin{array}{l}\text { Unterbrechung der vorderen Strukturen (knöchern } \\
\text { oder ligamentär) }\end{array}$ \\
\hline
\end{tabular}

\section{Neue AOSpine-Klassifikation der subaxialen HWS}

Die Klassifikation unterscheidet zwischen den prinzipiellen Entitäten A.0-4, B.1-3 und C sowie den zusätzlichen Qualitäten F.1-4, N.0-4 \& X \& + und M.1-4 (• Tab. 2-8 [4].

Die Buchstabenentsprechungen A-C stehen für die Morphologie des Bruches anhand der Entstehung ( $A=$ Kompression, $\mathrm{B}=$ Flexion/Distraktion, $\mathrm{C}=$ translatorische Verletzungen). F entspricht der Beteiligung der Facettengelenke, $\mathrm{N}$ dem neurologischen Status und $\mathrm{M}$ steht für sog. zusätzliche Modifikatoren.

Dabei werden die Verletzungen zuerst nach Höhe der Verletzung (C III-C VII) und deren Morphologie (A-C) beschrieben. Sind mehrere Level betroffen, wird zuerst die Höhe mit der schwerstwiegenden Verletzung beschrieben, gefolgt von der zweiten. Diese wird in Klammern gesetzt. Ebenso in Klammern werden die Subgruppen gesetzt: Facettengelenksverletzungen (falls unterschiedli- 


\section{AOSpine Subaxial Classification System}
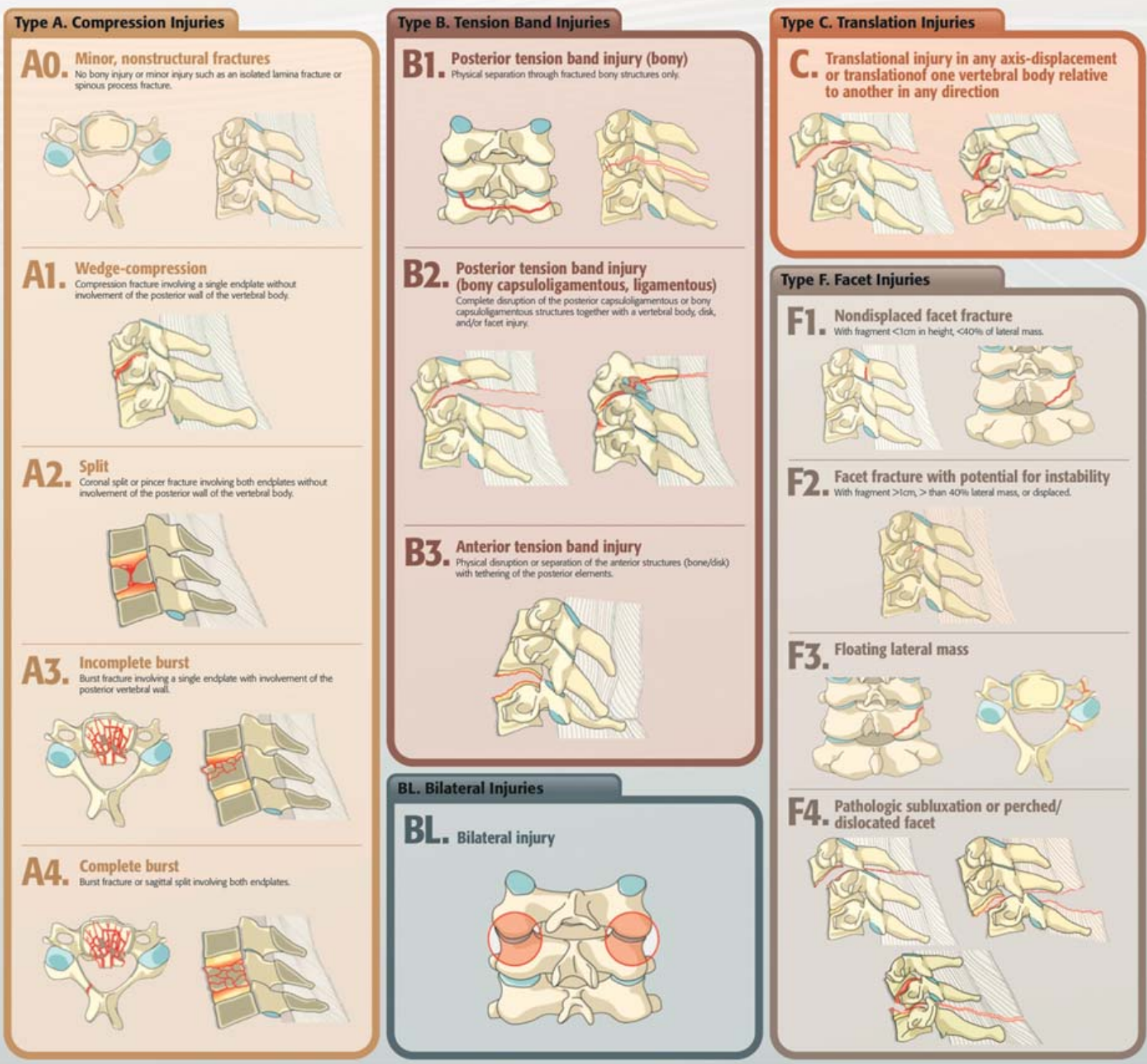

B2. Posterior tension band injury

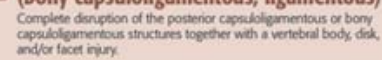

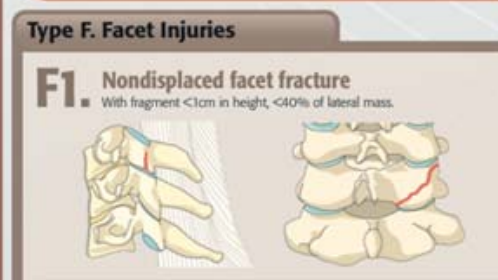

F2. Facet fracture with potential for instability
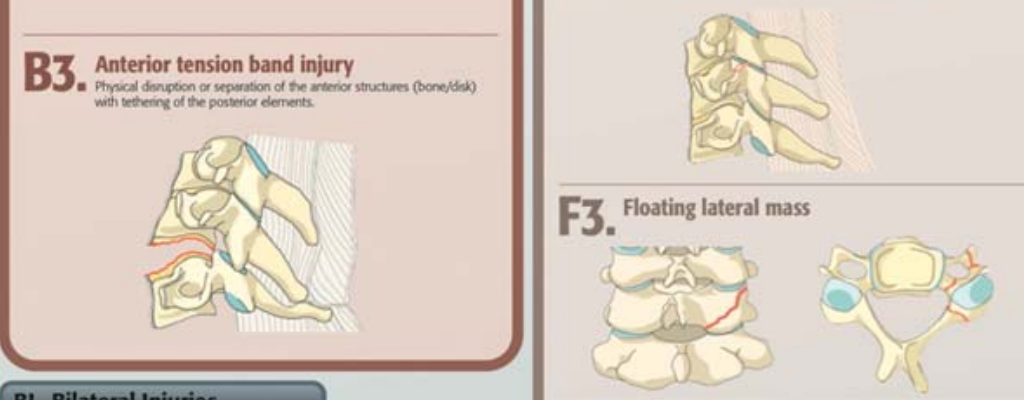

BL. Bilateral Injuries

B... Bilateral injury

\section{Pathologic subluxation or perched/}
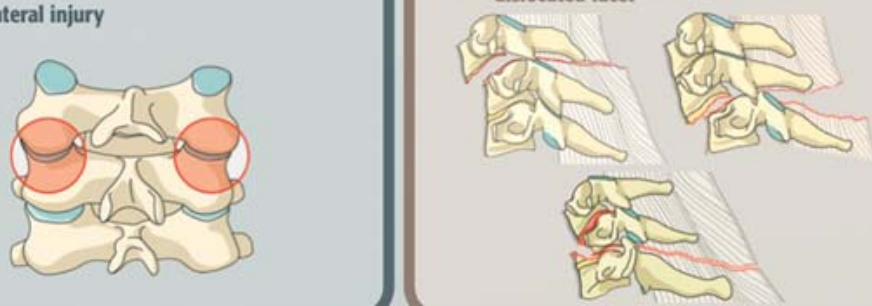

- Abb. 2 AOSpine-Klassifikation subaxiale HWS: Darstellung der einzelnen Verletzungsuntertypen. Copyright by AOSpine Interntional, Switzerland. Quelle: AOFoundation. AOSpine Subaxial Classification System. Im Internet: https://aospine.aofoundation.org/Structure/education/ online-education/classifications/Documents/aos_A1poster_SubaxialCS.pdf; Stand: 08.03.2018 [rerif].

ches Ausmaß auf einer Höhe wird rechts vor links benannt), neurologischer Status und Modifikatoren.

Gemäß dem folgenden Algorithmus wird die neue AOSpine-Klassifikation angewendet ( $\bullet$ Abb. 1):

Die einzelnen Untergruppen sind im Folgenden tabellarisch beschrieben und in $>$ Abb. 2 dargestellt:
Ein klinisches Beispiel ist in $>$ Abb. 3 illustriert.

\section{Prognose}

Zur Validierung der neuen AOSpine-Klassifikation wurde im Rahmen einer Kohortenstudie die Anwendbarkeit der neuen Klassifikation anhand radiologischer Befunde getestet. Hierfür wurden unabhängigen Spezialisten die Informationen über die neue Klassifikation im Sinne einer 
- Tab. 5 AOSpine-Klassifikation subaxiale HWS: Typ-CVerletzungen: translatorische Verletzungen.

\section{C translationale Verletzungen}

jegliche Form von translatorischer Verletzung, die in einem Versatz der Wirbelkörper in jeder Richtung zueinander resultiert

- Tab. 6 AOSpine-Klassifikation subaxiale HWS: Facettengelenksverletzungen.

\begin{tabular}{|c|c|}
\hline $\mathbf{F}$ & Facettengelenksverletzungen \\
\hline 1 & $\begin{array}{l}\text { nicht dislozierte Fraktur des superioren oder infe- } \\
\text { rioren Facettenanteils mit }<1 \mathrm{~cm} \text { Fragmentgröße } \\
\text { oder }<40 \% \text { Beteiligung der Massa lateralis }\end{array}$ \\
\hline 2 & $\begin{array}{l}\text { nicht dislozierte Fraktur des superioren oder infe- } \\
\text { rioren Facettenanteils mit }>1 \mathrm{~cm} \text { Fragmentgröße } \\
\text { oder }>40 \% \text { Beteiligung der Massa lateralis }\end{array}$ \\
\hline 3 & $\begin{array}{l}\text { diskonnektierte Massa lateralis mit Unterbrechung } \\
\text { von Pedikel und Lamina, unterbrochene Artikulati- } \\
\text { on der betroffenen Facette }\end{array}$ \\
\hline 4 & dislozierte, subluxierte oder luxierte Facette \\
\hline $\mathrm{BL}$ & beidseitige Verletzung \\
\hline
\end{tabular}

- Tab. 7 AOSpine-Klassifikation subaxiale HWS: neurologischer Status.

\begin{tabular}{|c|c|}
\hline $\mathbf{N}$ & neurologischer Status \\
\hline 0 & intakter neurologischer Status \\
\hline 1 & $\begin{array}{l}\text { vorübergehende neurologische Symptomatik, die } \\
\text { sich innerhalb } 24 \text { h vollständig zurückbildet }\end{array}$ \\
\hline 2 & Wurzelläsion \\
\hline 3 & inkomplette Querschnittläsion \\
\hline 4 & komplette Querschnittläsion \\
\hline$x$ & kein neurologischer Status beurteilbar \\
\hline+ & $\begin{array}{l}\text { fortschreitende Myelonkompression resultierend in } \\
\text { inkompletter Querschnittläsion, entspricht symp- } \\
\text { tomatisch N3 }\end{array}$ \\
\hline
\end{tabular}

- Tab. 8 AOSpine-Klassifikation subaxiale HWS: Modifikatoren.

\begin{tabular}{|c|l|}
\hline $\mathbf{M}$ & Modifikatoren \\
\hline 1 & $\begin{array}{l}\text { Verletzung der dorsalen, kapsuloligamentären } \\
\text { Strukturen ohne komplette Unterbrechung }\end{array}$ \\
\hline 2 & kritische Diskusherniation \\
\hline 3 & $\begin{array}{l}\text { Ankylose/metabolische Erkrankung (diffuse idio- } \\
\text { pathische skeletale Hyperostose, Spondylitis anky- } \\
\text { losans, OPLL, OLF) }\end{array}$ \\
\hline 4 & \begin{tabular}{l} 
Verletzung der A. vertebralis \\
\hline
\end{tabular} \\
\hline
\end{tabular}
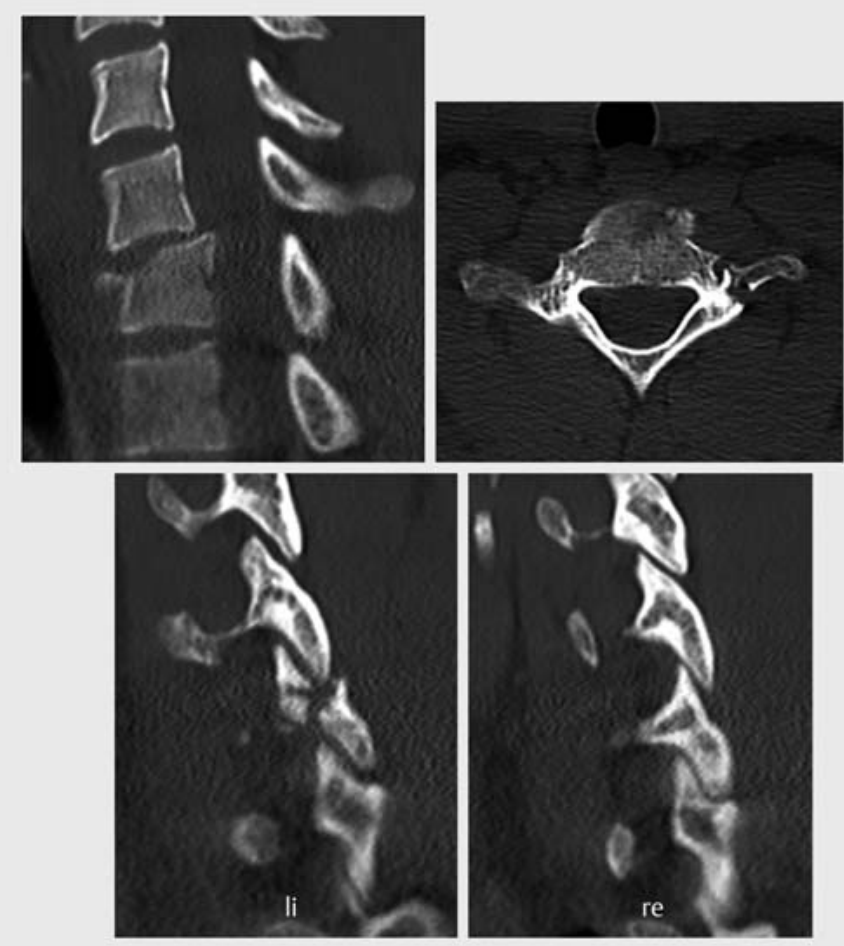

Abb. 3 Männlich, 23 Jahre alt, keine Neurologie: Darstellung der Klassifizierung.

Powerpoint-Präsentation übermittelt, anhand der Beschreibungen erfolgte dann die Klassifikation von 10 Frakturentitäten durch die Teilnehmer. Nach Rückübermittlung der Auswertungen an die Studienleitung und anschließender Korrektur erfolgte in einem zeitlichen Abstand von einem Monat die neuerliche Klassifikation von weiteren 30 Fällen anhand der neuen Methode.

Abschließend wurden intra- und interobservatorische Korrelationsanalysen anhand der Berechnung des sog. Kappa-Koeffizienten vorgenommen. Dabei wird der Grad der Übereinstimmung der in Zusammenhang gesetzten Beobachtungen mit einem metrischen Koeffizienten bewertet, der einem Wert zwischen 0 und 1 entsprechen kann. Je näher die Ausprägung des erhobenen Werts bei 1 liegt, desto höher ist der Grad der Korrelation zwischen den Beobachtungen. Im Rahmen der Arbeit wurde für die intra- und interobservatorischen Korrelationen ein Kappa-Koeffizient von 0,75 bzw. 0,64 erhoben, was laut Darstellung der Autoren einer hohen Übereinstimmung und somit einem positiven Ergebnis für die Anwendbarkeit des neuen Klassifikationssystems entspricht [2].

Die Verwendung im klinischen Alltag wird die Wertigkeit der neuen Klassifikation aufzeigen. 


\section{INFOBOX}

"AOSpine is a clinical division of the AO Foundation an independent medically guided nonprofit organization.

The AOSpine Knowledge Forums are pathology focused working groups acting on behalf of AOSpine in their domain of scientific expertise. Each forum consists of a steering committee of up to 10 international spine experts who meet on a regular basis to discuss research, assess the best evidence for current practices, and formulate clinical trials to advance spine care worldwide. Study support is provided directly through AOSpine's Research department and AO's Clinical Investigation and Documentation unit."

\section{Interessenkonflikt}

Die Autoren geben an, dass kein Interessenkonflikt besteht.

\section{Autorinnen/Autoren}

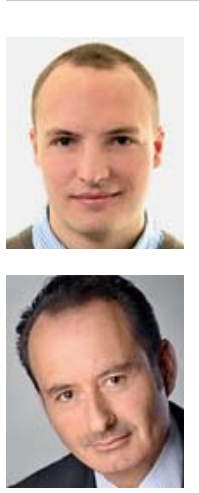

\section{Clemens Schopper}

Dr. med., Assistenzarzt, Klinik für Unfall-, Hand-, Plastische- und Wiederherstellungschirurgie, Universitätsklinikum Ulm

\section{Markus Schultheiss}

Prof. Dr. med., Geschäftsführender Oberarzt, Klinik für Unfall-, Hand-, Plastische- und Wiederherstellungschirurgie, Universitätsklinikum Ulm
Korrespondenzadresse

Prof. Dr. med. Markus Schultheiss

Klinik für Unfall-, Hand-, Plastische- und

Wiederherstellungschirurgie

Universitätsklinikum Ulm

Albert-Einstein-Allee 23

89081 Ulm

markus.schultheiss@uniklinik-ulm.de

Literatur

[1] Magerl F, Aebi M, Gertzbein SD et al. A comprehensive classification of thoracic and lumbar injuries. Eur Spine J 1994; 3: 184-201

[2] Vacarro AR, Koerner JD, Radcliff KE et al. AOSpine subaxial cervical spine injury classification system. Eur Spine J 2016; 25 : 2173-2184

[3] Schnake KJ, Schroeder GD, Vaccaro AR et al. AOSpine Classification Systems (subaxial, thoracolumbar). J Orthop Trauma 2017; 31 (Suppl. 4): S14-S23

[4] Patel AA, Dailey A, Brodke DS et al. Subaxial cervical spine trauma classification: the Subaxial Injury Classification system and case examples. Neurosurg Focus 2008; 25: E8

[5] Lee JY, Vaccaro AR, Lim MR et al. Thoracolumbar injury classification and severity score: a new paradigm for the treatment of thoracolumbar spine trauma. J Orthop Sci 2005; 10: 671-675

Bibliografie

DOI https://doi.org/10.1055/s-0044-101956

OP-JOURNAL 2018; 34: 112-116 @ Georg Thieme Verlag KG Stuttgart · New York ISSN 0178-1715 